\title{
Positive Emotions as a Potential Mediator of a Multi-Component Positive Psychology Intervention Aimed at Increasing Mental Well-Being and Resilience
}

\section{Tom Hendriks $^{1}$ (D) Marijke Schotanus-Dijkstra ${ }^{2}$ (D) Tobi Graafsma $^{3}$ (D) Ernst Bohlmeijer ${ }^{4}$. Joop de Jong ${ }^{5}$ (D)}

Accepted: 4 May 2020 / Published online: 12 May 2020

(C) Springer Nature Switzerland AG 2020

\begin{abstract}
We examined mediators of a multi-component positive psychology intervention for healthy employees. We analyzed data from a randomized controlled trial involving 158 participants who were assigned to a six-session intervention program or a wait-list control group. In total, 144 participants completed baseline and post-test assessment. The mediating effects of positive emotions and psychological flexibility on treatment outcomes mental well-being and resilience at post-test assessment were examined. We also examined the influence of a range of moderators on the relationship between the intervention and the outcomes. Mediator analyses showed that these improvements possibly and partially work through increasing positive emotions. Psychological flexibility could not be considered a mediator. However, due to inability to infer causality, definite conclusions on the mediating effect of positive emotions cannot be drawn. In relation to mental well-being, a significant moderating effect for ethnicity was found in the intervention group.
\end{abstract}

Keywords Mental well-being $\cdot$ Resilience $\cdot$ Mediators $\cdot$ Randomized controlled trial . Positive psychology

\section{Introduction}

Since the advent of the positive psychology movement, there has been a growing number of studies investigating the effectiveness of positive psychology interventions (PPIs) on mental well-being and mental disorders (Rusk and Waters 2013). PPIs are interventions aimed at increasing positive feelings, behaviors, and cognitions, using

Tom Hendriks

tom.hendriks@uvs.edu

Extended author information available on the last page of the article 
evidence-based pathways or strategies to increase well-being (Schueller et al. 2014; Schueller and Parks 2014). To date, several meta-analyses have examined the efficacy of PPIs. The reported effect sizes on mental well-being varied considerably, from small to large (Bolier et al. 2013; Chakhssi et al. 2018; Hendriks et al. 2018; Hendriks et al. 2019; Sin and Lyubomirsky 2009). This discrepancy can be attributed to the differences in included studied, for instance the larger the number of studies in the meta-analysis, the smaller the effect sizes on well-being. The meta-analysis by Sin and Lyubomirsky (2009) included randomized and quasi-experimental studies and reported a large effect size on well-being, whereas most other meta-analyses that included only randomized studies reported small to medium effects on well-being. Region of origin also has a moderating effect, with studies from non-western countries reporting larger effect sizes than studies from western countries (Hendriks et al. 2018; Hendriks et al. 2019). While many studies now have established the efficacy of PPIs, it is less clear what the working mechanisms of PPIs are. Several studies have explored the presence of mediators, i.e. variables that represent mechanisms or pathways through which an independent variable is able to influence a dependent variable (Barron and Kenny 1986). Studies that focused on the relationship between PPIs and mental well-being have identified the following factors as possible mediators: positive emotions ( $\mathrm{Li}$ et al. 2017; Schiffrin 2014; Schotanus-Dijkstra et al. 2019; Tugade and Fredrickson 2004; Vulpe and Dafinoiu 2012), empathy (Sandage and Worthington Jr. 2010), gratitude (Emmons and McCullough 2003), goal setting (Otto et al. 2016), positive thinking (Yu et al. 2015), self-efficacy (Toussaint et al. 2014), and psychological flexibility (Kashdan et al. 2006). In contrast to studies on well-being, to date, there have only been a few studies that examined the relationship between well-being interventions and resilience (Abbott et al. 2009; Robertson et al. 2015; Sood et al. 2011). These studies were conducted in western countries, and did not examine the role of mediators.

\subsection{Positive Emotions}

In this article, we firstly focus on positive emotions as a possible mediator, since several models have suggested that increasing positive emotions is central if positive changes in the ability to deal with adversities or mental wellbeing are to be attained. Building on the work of Isen 1984, Isen et al. 1985, Isen et al. 1987), Fredrickson (2001) argued that positive emotions expand people's attention and cognition, which enables creative problem solving and flexible thinking. Hence, positive emotions broaden a person's thought and action repertoire, which in their turn build durable, personal, physical, and psychological resources (Fredrickson 2004; Fredrickson and Branigan 2005). Other theoretical models have also identified positive emotions as a central element for positive change. For instance, the hedonic adaptation model (Sheldon and Lyubomirsky 2012), holds that a particular change in life, that is accompanied by the experience of positive emotions, will result in an initial boost in well-being. Through the occurrence of a number of positive events during a giving period (such as an intervention) well-being can be maintained. The positive activity model suggests that four particular elements of positive activities can increase well-being, namely (positive) emotions, thoughts, behaviors, and need satisfaction (Lyubomirsky and Layous 2013). Positive change can also be understood through the framework of the emotion regulation theory. This theory suggests different emotion regulation strategies that can 
increase positive emotion (Quoidbach et al. 2015). Lastly, the synergetic change model suggests that lasting changes in mental well-being depends on a complex, dynamic interaction between various domains of psycho-social functions (Rusk et al. 2018). The model covers five major domains, including positive emotions.

\subsection{Psychological Flexibility}

Psychological flexibility is another mechanism that may explain why positive changes during interventions are achieved, but which has gained lesser attention in the academic field compared to positive emotions. The concept of psychological flexibility has been studied for over 50 years, under a numerous different names including ego-resilience (Block 1961; Block and Block 2006), self- regulation (Carver and Scheier 2001), and response modulation (Patterson and Newman 1993). Kashdan and Rottenberg (2010, p. 2) describe psychological flexibility as a dynamic process involving: (1) adaptation to changing situational demands, (2) reconfiguring of mental resources, (3) shifting perspective, and (4) balancing competing desires, needs, and life domains. They argued that psychological flexibility is a key ingredient to psychological health, but that it has largely been neglected by (positive) psychologists as a cornerstone for daily well-being. Recently, Hinton and Kirmayer (2017, p. 3) have presented the flexibility hypothesis of healing. According to this theory flexibility is "the ability to consider different actions, adopt multiple cognitive frames, or "mindsets", explore possibilities, and experience diverse modes of being-in-world". Drawing on their work in the field of anthropology and cultural psychiatry, Hinton and Kirmayer (2016) argue that all healing practices (western psychotherapy and indigenous practices alike), induce positive psychological states marked by cognitive and emotional flexibility or an increased ability to shift cognitive sets. Thus, (psychological) flexibility is a general mechanism that may also explain the working of PPIs. It has been suggested that positive emotions may build psychological flexibility, and that psychological flexibility in its turn generates positive emotions (Cohn 2008; Ong et al. 2006; Tugade and Fredrickson 2004; Vulpe and Dafinoiu 2012). For example, a study among US college students following the September 11th terrorist attacks, demonstrated that students with high levels of ego resilience (i.e. psychological flexibility) were better to rebound from adversity, and the relation was mediated by positive emotions (Fredrickson et al. 2003). In view of the relationship between positive emotions and psychogical flexibility, we chose psychological flexibility as a second major mediator.

\subsection{Present Study}

We conducted a randomized controlled trial among a group of employees with a multiethic background in Paramaribo, Suriname (Hendriks et al. 2020). The current study examines mediators of a multi-component positive psychology intervention. For practical reasons (e.g. limited attention span of the participants) we had to limit the number of possible mediators we could measure in our study. We focused on two possible mediators in the development of well-being and resilience, namely positive emotions and psychological flexibility. Although there is a lack of literature, available studies suggest positive emotions as a possible mechanism for change, as described earlier in this paper. A qualitative study we conducted prior to the intervention, suggested that 
acceptance is a major character strength among the Surinamese (Hendriks et al. 2017), and acceptance is one of the core processes to develop psychological flexibility (Hayes 2006). Also the ability to adapt to new circumstances was often mentioned as a reason why Surinamese have been able to deal with economic and political adversities in recent history. This led us to consider psychological flexibility as a possible mechanism. Both potential mediators are linked to theoretical frameworks that attempt to explain how and why PPIs can increase mental well-being. We hypothesize that both positive emotions and psychological flexibility mediate the relation between the intervention and the outcomes. In addition, we will also investigate moderators, i.e. variables on which the effectiveness of any given treatment may depend (Kraemer et al. 2002).

\section{Methods}

\subsection{Participants and Procedure}

Employees from three companies in Paramaribo, Suriname were recruited. Potential participants were screened by the human resource managers of the companies. The inclusion criteria were as follows: (1) age between 18 and 60 years; (2) participants had sufficient knowledge of the Dutch language to fill out questionnaires, read the training manual, and participate in written exercises; (3) willing to participate in a weekly twohour program for six consecutive weeks. The study was a single-blinded parallel randomized controlled trial with an active intervention group and a waiting list control group. After screening, participants were randomly assigned to either the intervention or the control condition, using the online program research randomizer (https://www. randomizer.org). The assessment of the outcomes was done through self-report measures (paper-and-pencil tests). Baseline assessment was administered during an initial informational session with all participants (T0), after which the participants received the envelopes with the allocation details. One week after the initial session, the official training program started for the intervention group. In total six sessions were conducted in six consecutive weeks. After six sessions, post-test assessment was conducted for the intervention group and the waiting list group (T1). After the intervention group finished in the program, the participants in the waiting list condition started the intervention. Follow-up measures (T2) were conducted at three months after the end of the training sessions. Figure 1 provides an overview of the flow of participants.

\subsection{The Intervention}

The intervention program was named Strong Minds Suriname (SMS) and consisted of a series of activities that involved exploring one's positive emotions, engaging in new activities, reframing negative thoughts and adopting a positive and adaptive mind-set. Six weekly sessions were conducted and each session had a specific theme, namely (1) gratitude; (2) positive emotions; (3) discovering strengths; (4) goal setting; (5) positive thinking; (6) letting go: forgiveness, surrendering and prayer. Except for the first informational sessions, each session started with psycho-education, followed by three or four positive activities. The exercises were conducted by local coaches who prior to the intervention received training. Exercises included a variety of prototypical positive 


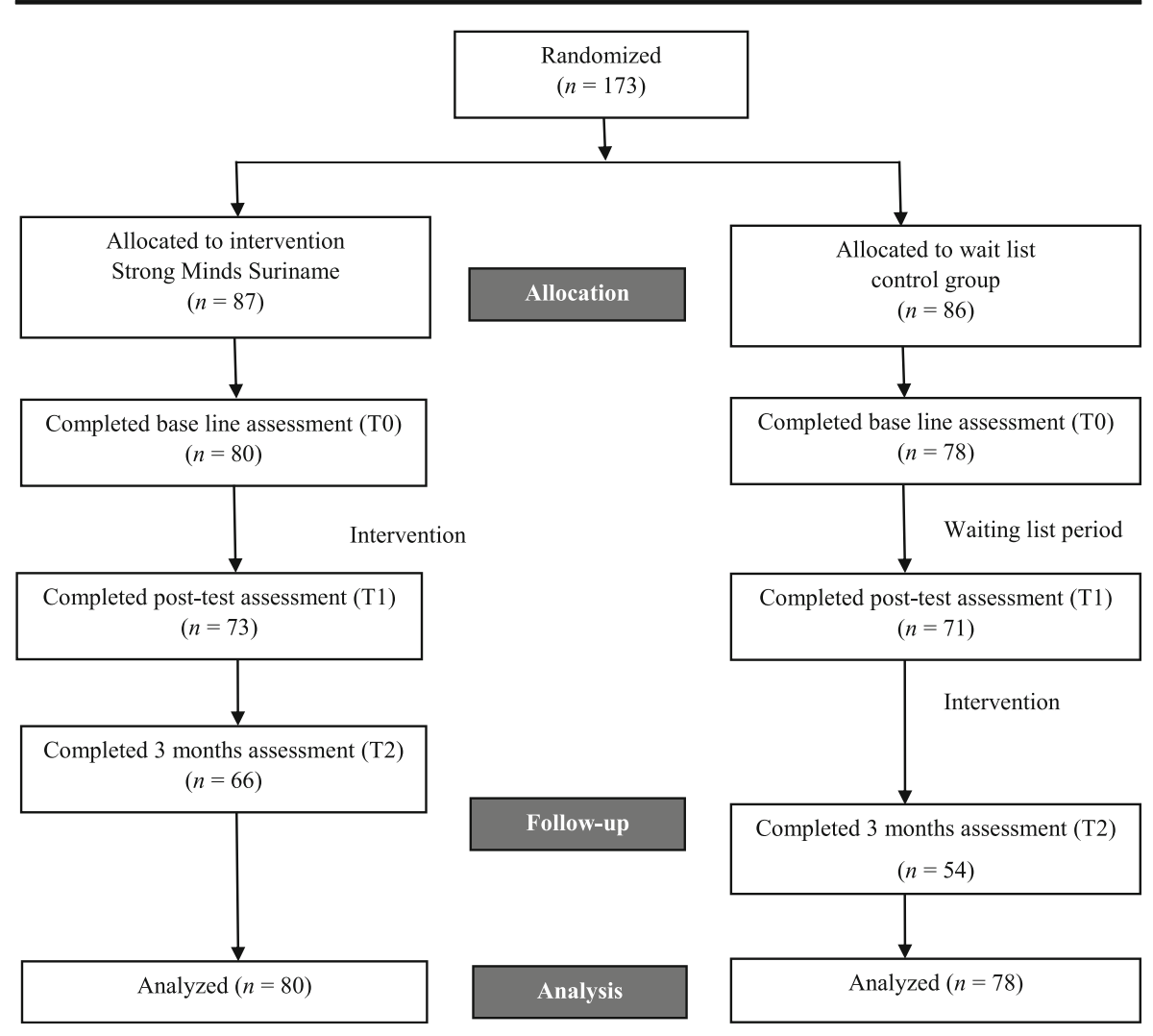

Fig. 1 Flow chart of the participants

psychology activities, including writing a gratitude letter (Seligman et al. 2006), performing acts of kindness (Buchanan and Bardi 2010), identifying and using strengths (Proyer et al. 2015), positive goal setting (Bolier et al. 2010), and practicing forgiveness (Akhtar and Barlow 2016). Breathing exercises, expressing gratitude through prayer, and silence meditation were also practiced. Voluntary homework assignments included keeping a gratitude journal and paying a gratitude visit. Homework compliance was checked by the coaches at the beginning of the session following the assignments, but not registered to avoid any distrust towards the coaches or fear for repercussions. The duration per module was two to three hours, including a break. Cultural adaptation was conducted according to a three phased process, following guidelines for cultural adaptation as described by Domenech-Rodriguez and Wieling (2004) and guidelines for the implementation of cultural sensitive cognitive behavioral therapies (Hinton and Jalal 2014; Hinton et al. 2013; Hinton et al. 2012). During the first phase, focus group meetings with human resource management representatives of the participating companies were conducted, to select topics on well-being and resilience that seemed relevant to the Surinamese context. During the second phase the content of the original SRP training was adapted and new content was added. Additional focus group meetings focused on reviewing the content, semantic, conceptual, and technical equivalence of the program and the measure instruments. During the third stage of the adaptation process, the intervention was tested on a group of eight 
employees from the participating companies. These employees were also trained as coaches for the intervention. During the intervention the coaches were observed for adherence to the training protocol. On the basis of their feedback, the training manual and exercises were adjusted and the final version of the training was developed. For a detailed description of the content of the intervention, see Appendix 1.

\subsection{Outcome Measures}

Mental well-being was measured with the Mental Health Continuum-Short Form (MHC-SF) (Keyes 2005), a 14-item questionnaire that measures emotional well-being, social well-being, and psychological well-being. The three-factor structure of the MHC-SF and its reliability and construct validity have been validated in a range of studies from non-western countries including Argentina (Perugini et al. 2017), Iran (Joshanloo 2016), Korea (Lim 2014), and South Africa (Keyes et al. 2008). We used the validated Dutch version, for which the structure, the reliability, and the discriminant and construct validity also have been validated (Lamers et al. 2011). Participants had to indicate the frequency of a range of experienced emotions over the past four weeks. Item scores range from "never" (0) to "(almost) always" (5), with a higher mean score indicating a higher level of well-being. Its Cronbach's alpha in the present study was .89 at pre-test, .92 at post-test, and .94 at follow-up assessment. Resilience was measured with the Dutch Resilience Scale (RS-nl) (Portzky et al. 2010). This scale is a Dutch adaptation of the Wagnild and Young Resilience Scale (Wagnild and Young 1993). The original version of the RS has been used in many non-western countries and validation studies showed positive results on a variety of psychometric properties, for example among populations in Brazil (Pesce et al. 2005), Mexico (Heilemann et al. 2003), Nigeria (Abiola and Udofia 2011), Sri Lanka (Munasinge 2012), and China and Taiwan (Yang et al. 2012). Although a study on the validity of the Dutch version of RS did not find strong evidence for a solid five factor structure, the Dutch version appears to be valid and reliable (Portzky et al. 2010). The scale consists of 25 items that measures two dimensions of resilience: personal competence and acceptance of self and life. It uses a 4-point Likert scale with two anchoring statements from 'strongly disagree' (1) to 'strongly agree' (4), with a higher mean score indicating a higher level of resilience. Its Cronbach's alpha in the present study was .90 at pre-test, .92 at posttest, and .93 at follow-up assessment.

\subsection{Mediator Measures}

Positive emotions were measured with the Positive Affect subscale of the Positive and Negative Affect Schedule (PANAS), a 20-item scale that measures two opposites of mood (Watson et al. 1988). The PANAS appears to have adequate psychometric properties (Crawford and Henry 2004). It is used widely across the globe and studies show that the questionnaire is non-invariant between respondents from a collectivistic cultural context (non-western countries) and those from individualistic cultural context (western countries) (Weidong et al. 2004; Lee et al. 2020). We used the Dutch version of the PANAS, for which the psychometric properties also appear to be solid (Engelen et al. 2006). Items on the PANAS are rated on a five-point scale ranging from "little, or not very much" (0) to "very much" (10), with a higher score indicating a higher level of 
positive or negative feelings and thoughts. Cronbach's alpha for positive affect in the present study was .86 at pretest, .86 at post-test, and .90 at follow-up assessment. Cronbach's alpha for negative affect was .84 at pretest, .86 at post-test, and .88 at follow-up assessment. Psychological flexibility was measured with the Psychological Flexibility Questionnaire (PFQ). The PFQ originally is a 20-item questionnaire that measures five factors related to psychological flexibility: (1) positive perception of change; (2) characterization of the self as flexible; (3) self-characterization as open and innovative; (4) a perception of reality as dynamic and changing; (5) a perception of reality as multi-faceted. The PFQ has not widely been used, and only one study demonstrated its convergent validity and reliability (Ben-Itzhak et al. 2014). We translated the questions to Dutch and selected all 13 questions of the first three factors. We did not include questions pertaining to the last two factors. This was done on the basis of feedback from a focus group meeting of psychologists in the context of the cultural adaptation process. The questions from the PFQ on reality perception were considered too complex and abstract for our target population (lower educated employees). Items of the PFQ are rated on a 6-point Likert-type scale from "not at all" (1) to "very much" (6), with a higher score indicating greater psychological flexibility. Its Cronbach's alpha in the present study was .87 at pre-test, .92 at post-test, and .95 at follow-up assessments. These findings are comparable to the reliability of the original scale that reported a Cronbach's alpha coefficient of .85 .

\subsection{Moderator Measures}

The following demographic data from the participants were gathered: age, gender, ethnicity (Afro-Surinamese, Hindustani, Javanese, Mixed, Amerindian, other), religion (Christian, Islam, Hinduism, other, no religion), education level (lower, middle, or higher), and company of employment (Intermed, Surinaamse Postspaarbank or MultiElectrical Systems). Levels of financial distress at baseline were measured, using the InCharge Financial Distress/FinancialWell-Being Scale (IFDFW), an eight item scale that measures levels of financial distress or financial well-being (Prawitz et al. 2006). Levels were classified as either low, moderate, or high. Finally, the adherence of the intervention group to the program was measured. Attendance to six sessions was coded as a high level of adherence, attendance to four or five sessions as a moderate level of adherence, and attendance to one, two, or three sessions as a low level of adherence.

\subsection{Statistical Analyses}

Missing data at baseline and post-test were imputed, using the estimation maximization (EM) algorithm (Dempster et al. 1977). Mediator analyses were performed according to the procedures as described by Preacher and Hayes (2008), using the PROCESS tool, version 3.0 (Hayes 2012). Multiple mediator analyses were performed, by entering the core processes (positive emotions and psychological flexibility) simultaneously in the regression models, using model 4 . In the analyses, $\mathrm{X}$ is the intervention condition (coded 1 for the intervention group and 0 for the waiting list control group). $\mathrm{Y}$ is the change in outcomes (increases in well-being and resilience), from T1-T0, and M is the possible mechanism of change from T1-T0 (increases in positive emotions and flexibility). For each path, unstandardized regression coefficients were calculated. Path $a$ 
represents the effect of the intervention (X) on the mediator $(\mathrm{M})$, and path $b$ represents the effect of the mediators $(\mathrm{M})$ on the outcomes $(\mathrm{Y})$. Path $c$ represents the total effect of the intervention $(\mathrm{X})$ on the outcome $(\mathrm{Y})$ and path $c$ ' is the direct effect of the intervention $(\mathrm{X})$ on the outcome $(\mathrm{Y})$, while partialling out the effect of the mediator (M). The indirect effect of $\mathrm{X}$ on $\mathrm{Y}$ through $\mathrm{M}$ is calculated as the product of $a$ and $b$ $(a b)$, of which the bias corrected (BC) $95 \%$ were based on 5000 bootstrap samples. Estimates are statistically significant at $p<0.05$, or if the $95 \%$ CI did not contain zero (Preacher and Hayes 2008). The moderator analyses were also conducted using the PROCESS tool. Results were computed using model 1, with a 95\% CI, using 5000 bootstrap samples. In the analyses resilience and mental well-being at post-test assessment (T1) were the dependent variables (Y) and resilience and mental well-being at baseline assessment (T0) were the independent variables (X). The potential moderators were separately entered as moderator variables $(\mathrm{W})$.

\section{Results}

\subsection{Demographics}

The mean age of the participants was 36.3 years $(\mathrm{SD}=9.6$ years) and $60 \%$ of the participants was female. The largest ethnic group was the Javanese (42\%), followed by the Hindustani $(25 \%)$, and the Afro-Surinamese group (15\%). Participants of mixed origins accounted for $17 \%$ of the population. The main religious backgrounds were Christianity (42\%), Islam (30\%), and Hinduism (18\%). The majority of participants had a lower $(46 \%)$ or intermediate educational level $(42 \%)$. The Intermed group (private healthcare company) provided $44 \%$ of the participants, the Surinaamse Postspaarbank (savings bank) 29\%, and Multi Electrical Systems (construction company) $27 \%$.

\subsection{Drop out}

Initially, 173 participants were randomized. However, two days before the start of program participation of fifteen employees from one company was cancelled, due to unexpected changes in the working schedule. We defined drop-outs as participants who completed the baseline assessment but who did not complete post-test and/or follow-up assessments. In total, 158 participants completed the baseline assessment (T0), 144 (91\%) completed the post-test assessment (T1) at seven weeks, and $120(76 \%)$ the three-month follow-up assessment (T2). There were 14 drop-outs (9\%) between pretest and post-test. Between post-test and follow-up assessment 24 participants dropped out $(14 \%)$. The difference between drop-outs in the intervention group and waiting list group was not significant. At the post-test assessment (T1), there was a significant difference in age between drop-outs and completers $(\mathrm{M}=46.1$ vs $35.4, \mathrm{~F}(1-156)=$ $1.15, p<0.001)$. In addition, we found that the average education level and income of drop-outs was significantly lower. This indicates that employees with a low educational level and low income were more likely to drop-out. At follow-up, we found no significant differences between drop-outs and completers on any measured demographics. Further analysis showed that drop-outs significantly scored higher on 
resilience at pre-test than completer. This indicates that people with a higher level of resilience were more inclined to drop-out.

\subsection{Bivariate Correlations}

Table 1 displays the bivariate correlations between mental well-being (that include the subscales of the MHC-SF), resilience and the potential mediators. We found that all correlations were significant and ranged from .29 to .89. Most correlations were moderate to strong. High correlations were found between all different outcomes of mental well-being. The correlations between the scales measuring resilience and the potential mediators were higher than the correlations between the subscales of mental well-being and the potential mediators. A low score was found between positive affect and emotional well-being.

\subsection{Multiple Mediation Models}

We performed multiple mediation analyses. Table 2 shows the results from the mediation analysis on mental well-being and resilience.

Figures 2 and 3 show the unstandardized regression coefficients on changes in mental well-being and resilience, respectively. Coefficients of the a-paths on mental well-being and resilience were significant for positive emotions $(p<.01)$ but not for psychological flexibility $(p=.13)$. This implies that the intervention led to increased levels of positive emotions, but not to an increase in psychological flexibility. All bpaths were significant and show that positive emotions predicted mental well-being (moderate effect) and resilience (small effect), and psychological flexibility predicted mental well-being (large effect) and resilience (small effect). The intervention was effective in increasing mental well-being and resilience, and this was still the case when including the mediators (c'). The effect of including positive emotions and psychological flexibility as mediators for mental well-being was larger, whereas the effect of these mediators on resilience was small. The BC 95\% CI of the specific indirect effect for positive emotions for well-being did not contain zero $(a b=.08, \mathrm{BC} 95 \% \mathrm{CI}=.01$ to $.17)$, while it did contain zero for psychological flexibility $(a b=.10, \mathrm{BC} 95 \% \mathrm{CI}=-.01$ to .23) Regarding resilience, the BC $95 \% \mathrm{CI}$ of the specific indirect effect for positive

Table 1 Bivariate correlations between potential mediators and outcome measures at baseline

(1)

(2)

(3)

(4)

(5)

(6)

(7)
(1) MHC-SF
(2) MHC-SF EMO
(3) MHC-SF SOC
(4) MHC-SF PSY
(5) RS-nl
(6) PANAS-PA
(7) PFQ

Mental well-being

1

Emotional well-being

$.78^{*} \quad 1$

Social well-being

$.88^{*} \quad .56 * \quad 1$

Psychological well-being

$.89 *$

$.60^{*}$

Resilience

$.48 *$

$.40^{*}$

$.62 * \quad 1$

$.48 *$

$.33^{*}$

$.29 *$

.54

Positive affect

.52

$.30 *$

$.36 *$

Psychological flexibility

.40

$* p<.001$ 
Table 2 Results from the multiple mediation analysis for effects of the intervention (Strong Minds Suriname) versus wait-list group (T1-T0) on mental well-being and resilience

\begin{tabular}{|c|c|c|c|c|c|}
\hline Mediators & $\mathrm{a}$ & $\mathrm{b}$ & Total effect $\mathrm{c}$ & Direct effect c' & Indirect effect $\mathrm{a} \times \mathrm{b}(95 \% \mathrm{CI}) \mathrm{a}$ \\
\hline \multicolumn{6}{|l|}{ Mental well-being } \\
\hline Positive emotions & $.21 * *$ & $.37 *$ & $.62 * * *$ & $.45 * * *$ & $.08(.01-.17)$ \\
\hline Psychological flexibility & $.13 \mathrm{~ns}$ & $.76^{* * *}$ & $.62 * * *$ & $.45^{* * * *}$ & $.10(-.01-.23)$ \\
\hline \multicolumn{6}{|l|}{ Resilience } \\
\hline Positive emotions & $.21^{* *}$ & $.18 * *$ & $.23 * * *$ & $.18^{* * * *}$ & $.04(.01-.09)$ \\
\hline Psychological flexibility & $.13 \mathrm{~ns}$ & $.13 *$ & $.23 * * *$ & $.18 * * *$ & $.02(-.01-.06)$ \\
\hline
\end{tabular}

Notes: $* \mathrm{p}<.05, * * \mathrm{p}<.01, * * * \mathrm{p}<.001, \mathrm{~ns}=$ not significant

emotions also did not contain zero $(a b=.04, \mathrm{BC} 95 \% \mathrm{CI}=.01$ to .09$)$, while it did contain zero for psychological flexibility $(a b=.02, \mathrm{BC} 95 \% \mathrm{CI}=-.01$ to .06$)$. This suggests that positive emotions are a mediator for well-being and resilience, whereas psychological flexibility is not a mediator. The total model explained $12 \%$ of the variance in well-being and $26 \%$ of the variance in resilience.

\subsection{Moderator Analysis}

We found no significant interaction effects for any of the moderators on resilience at post-test assessment. For mental well-being, a significant effect was found for ethnicity in the intervention group $(p=0.03)$. This suggests that people from the Javanese ethnic group benefited more from the intervention than Hindustani or people from mixed ethnicity. The moderator $x$ group interaction effects on mental well-being and resilience are shown in Appendix 2

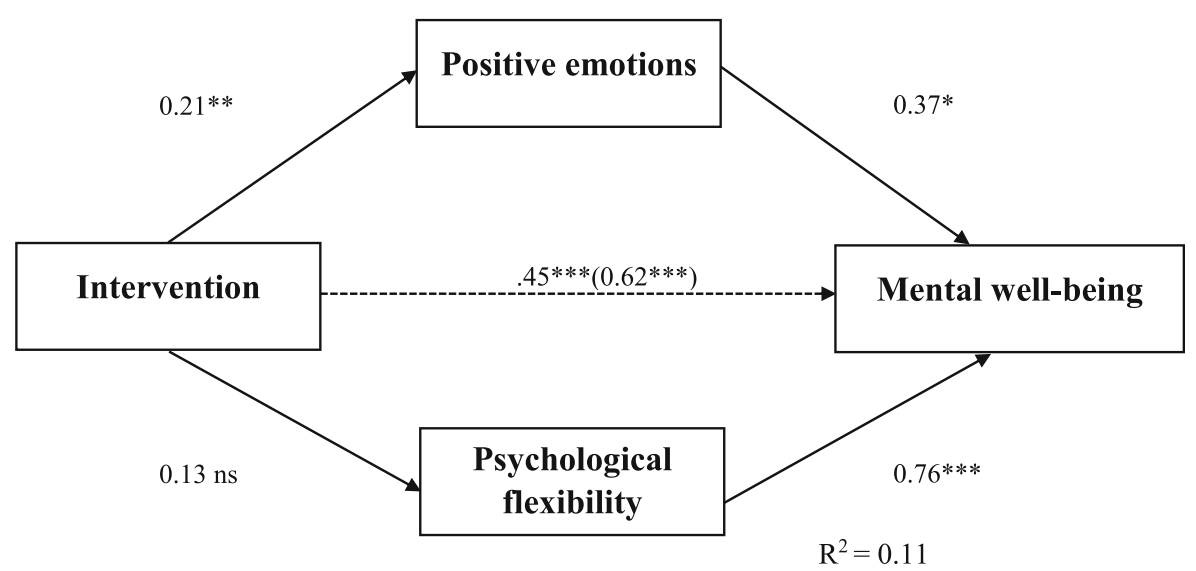

Fig. 2 Multiple mediation positive emotions and psychological flexibility as mediators of the intervention group versus wait-list group (T0-T1) on mental well-being Total effect (c-path) is given in parentheses. * $p$ $<.05, * * p<.01, * * * p<.001, \mathrm{~ns}=$ not significant 


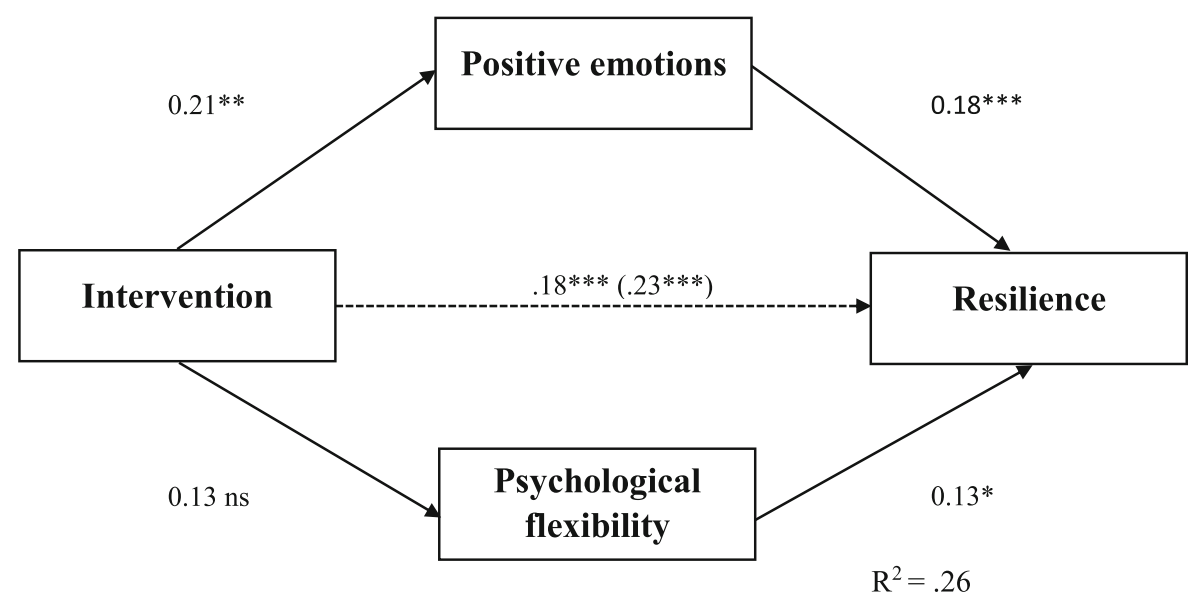

Fig. 3 Multiple mediation positive emotions and psychological flexibility as mediators of the intervention group versus wait-list group (T0-T1) on resilience Total effect (c-path) is given in parentheses. *p $<.05$, **p $<.01, * * * \mathrm{p}<.001, \mathrm{~ns}=$ not significant

\section{Discussion}

The aim of this study was to investigate possible mechanisms of change of a culturally adapted multi-component positive psychology intervention. We hypothesized that positive emotions and psychological flexibility mediate the relationship between the intervention program and mental well-being and resilience. The intervention used in this study has previously shown to increasing well-being and resilience with large effect sizes for mental well-being $(d=0.62,95 \% \mathrm{CI}=0.30-0.94)$ and resilience $(d=.76,95 \% \mathrm{CI}=.43-1.09)$ (Hendriks et al. 2020). These results were in line with earlier studies that reported improvements on mental well-being for MPPIs (Carr et al. 2016; Cullen et al. 2015; Feicht et al. 2013; Nikrahan et al. 2016; Proyer et al. 2016). Multiple mediation analyses in the current study demonstrated that the intervention led to increased levels of mental well-being and resilience at post-test, through its effect on positive emotions. However, in the multiple mediation models, the percentages of the variance in the outcomes were small, $11 \%$ in mental well-being and $26 \%$ for resilience, respectively. Therefore, we conclude that the mediating role of positive emotions is small. While several correlational studies have reported the meditating role of positive emotions on well-being and resilience (Schiffrin 2014; Tugade and Fredrickson 2004; Vulpe and Dafinoiu 2012), there is a lack of causal studies examining possible mediators in PPIs. Recently, a RCT among 275 adults with suboptimal mental health demonstrated that positive emotions, along with the use of strengths, optimism, selfcompassion, resilience and positive relations mediated the development of mental wellbeing, during an 8-week multi-component PPI (Schotanus-Dijkstra et al. 2019). However, positive relations, self-compassion and optimism were the strongest predictors of the efficacy of the intervention on mental well-being, anxiety and depression, and the mediating effect of positive emotions were small. This finding is in line with the results from our mediator analysis, suggesting a limited role for positive emotions as a possible mechanism for change through a multi-component PPI. 
Our study did not find support for the hypothesis that psychological flexibility played a mediating role between the intervention and well-being and resilience. This outcome contrasts with findings from other studies that identified the mediating role of psychological flexibility on mental well-being (Fledderus et al. 2013; Kashdan et al. 2006; Wicksell et al. 2010) and resilience (Ong et al. 2006; Tugade and Fredrickson 2004; Vulpe and Dafinoiu 2012). Our study also demonstrated that the intervention did not increase psychological flexibility. This could be explained by the following. First, the intervention only contained two sessions that each featured one exercise aimed at stimulating mental flexibility, namely negative changing beliefs and practicing forgiveness (see Appendix 1). In contrast, the expression of positive emotions was practiced in at least eight exercises throughout the intervention. In addition, no psycho-education on the importance of psychological flexibility in relation to mental well-being or resilience was given, whereas the benefits of positive emotions were addressed in two psycho-educative sections in the program. Second, perhaps increases in flexibility were not possible due to a ceiling effect. During the period prior to our study, participants had to deal with high inflation rates, 55\% in 2016 and $22 \%$ in 2017 (KNOEMA 2018). The majority of the participants $(65 \%)$ indicated that they earned less than 270 US dollars per month. It is very likely that in order to adapt to the financial changes, flexibility was already at a high level. In addition, it is possible the cultural background of the participants is responsible for already high levels of flexibility. In addition, the majority of the participants in our study also had an East-Asian ethnic background (67\%). People from East-Asian cultures tend to value low arousal emotions (e.g. calmness, acceptance) more than people from the West (Tsai et al. 2006) and emphasize adjusting one's needs to those of their social environment (Shin and Lyubomirsky 2017), which requires psychological flexibility. Third, we used the PFQ questionnaire to measure psychological flexibility, and this instrument was not validated among the Surinamese population. During the cultural adaptation process of the intervention, seven questions were omitted from the original questionnaire, based on feedback of a focus group of Surinamese psychologists. This was due to the complexity of the questions, in view of our participants who for a large part had a low educational level. Perhaps the remaining were still complex, which could have led to extreme response style bias (He et al. 2014).

Moderator analyses only identified the variable ethnicity as a significant moderator in the relation between the intervention and mental well-being, suggesting that people from the Javanese ethnic group benefited more from the intervention than Hindustani or people from mixed ethnicity. This could be explained by the fact that the main trainer of the intervention was from Javanese descent. As suggested by the common factor theory (Frank et al. 1991), the relation between a therapist and a patient, is one of four main factors that contribute to the efficacy of an intervention (Colijn et al. 2009). We believe that this model is also applicable outside the realm of psychotherapy, i.e. to the relation between a trainer and participants in any intervention. In our study, the main trainer not only had a Javanese ethnic background, but was also the CEO of one of the participating companies. He served as a positive role model in the Javanese community in particular, and this could have influenced engagement and commitment among Javanese participants. 
This study has several limitations. First, we did not establish a timeline for the mediator and the outcomes, meaning that we did not measure positive emotions before resilience and mental well-being. According to Kazdin (2007, 2009), this is an essential requirement for demonstrating the effect of a mediator. Therefore, we cannot ensure that the level of positive emotions changed before resilience and mental well-being changed, so a definite causal relationship between the mediator and the outcome cannot be established. It is possible that resilience and mental well-being changed prior to the change in positive emotions. As Kazdin (2007, 2009) pointed out, a timeline problem is present in the vast majority of literature that examined mediators of psychotherapy. We found this also to be the case in positive psychology literature. Therefore, we recommend that studies that attempt to discover causal effects for mediators in PPIs establish a clear timeline, i.e. the measurement of the potential mediators should be conducted prior to the outcomes.

Second, we limited our study to two possible mediators. Focus groups meetings with HRM representatives of the participating companies and psychologists brought forth the advice to limit the number of questionnaires/items, in light of the low educational level of the participants and possible fatigue. Therefore, we were limited in the number of constructs we could measure. There are other processes that could play a mediating role in the development of resilience and well-being in Suriname. For example, strengths and virtues that are related to resilience, according to a previously conducted quantitative study in Suriname, namely religiousness, hope, harmony, acceptance and perseverance (Hendriks et al. 2017). Several theoretical frameworks and models have suggested other possible mechanisms for positive change. We recommend that future mediator studies focus on examining and operationalizing the mechanism for change that are presented in specific/such models. In particular, the Synergetic Change Model (SCM) (Rusk et al. 2018) is the most elaborate model today. The five domains of the SCM may correspond to specific mediators. Emotions is one of the major domains, and as described in this article, several studies have indeed identified positive emotions as mediators. Other mechanisms may correspond to the different domains of the SCM. For example, change in character strengths and virtues, as conceptualized by Values-In-Action (VIA) strengths classification system (Proctor et al. 2011), could be a possible mechanism, and is related to the domain 'virtues and relationships' of the SCM.

Third, we did not study the effects of demand characteristics. We could not disguise the purpose of the intervention, which was to increase resilience and well-being of the participants. However, several measures were taking to minimize possible effects of demand characteristics. The coaches who delivered the program to their co-workers were instructed not share their experiences with the participant outside the program during the trial. Participants in the intervention group were also instructed not to share experiences (including exercises) with colleagues that were in the waiting list condition. In addition, two researchers were present at the training, but interpersonal contact was minimalized. Finally, the intervention group and the waiting list group received the training under similar circumstances (e.g. same location, same trainers, on the same days and times, same training materials). 
Fourth, we used self-report questionnaires in Dutch, and these questionnaires were not validated within the local context. Although Dutch is the official language in Suriname, the spoken language during the intervention was mostly Sranang Tongo, an English-based creole language. Some questions in the instruments of measure may have been too complicated for participants with a limited Dutch language comprehension, and this may have biased the outcomes. This could, for example, lead to extreme or midpoint response style bias (He and van de Vijver 2012), or acquiescence bias which is more likely to occur among people with low socioeconomic status in collectivistic cultures (Harzing 2006). In order to limit possible bias, the questionnaires were checked for content, semantic, and conceptual equivalence, and were tested among a small group of employees prior to the intervention. During the assessment, participants could ask for assistance from local coaches, if they had difficulties understanding questions. Despite these measures, bias still may have played a role. For future research we recommend measuring outcomes in non-Western countries should preferable be done using questionnaires that are validated in the corresponding country. If this is not possible due to financial or other limitations, questionnaires should be at least checked for content, semantic and conceptual equivalence.

\section{Conclusion}

The past decade there has been an increasing amount of randomized studies have demonstrate the positive effects of positive psychology interventions (PPI) on mental well-being. However, few studies have focused on the mechanisms that may explain why positive changes are reported. To fill this gap in the research, we examined possible mediators of a cultural adapted multi-component PPI that was effective in increasing resilience and mental well-being among employees in Suriname (Hendriks et al. 2020). Mediator analyses showed that these improvements possibly and partially work through increasing positive emotions. This adds to the evidence that increasing positive emotions contributes to increased wellbeing. However, due to the absence of a timeline during measurement definite conclusions on the mediating effect of positive emotions cannot be draw. We did not find a possible mediating effect for psychological flexibility, perhaps because the intervention did not include sufficient exercises that focused on increasing this outcome. Future studies should investigate other possible mechanisms for lasting positive change, in order to develop the most efficacious combinations of components within multi-component PPIs that take into account the cultural background of the participants in the intervention.

Funding Information The study was funded by the University of Amsterdam and sponsored by the following participating Surinamese companies: Multi Electronic System N.V., Surinaamse Postspaarbank N.V., and the InterMed Group. Except for Wantley Sardjo, the CEO of Multi Electrical System N.V., and coauthor in this study, funders had no role in, or control over the collection, management, analysis, interpretation, and publication of the data.

Competing Interests The authors declare that they have no competing interests. 


\section{Appendix 1: Overview of the sessions and activities of the Strong Minds Suriname program}

Table 3 Overview of the sessions and activities of the Strong Minds Suriname program

\begin{tabular}{|c|c|c|}
\hline \# & Sessions & Positive activities \\
\hline 1 & $\begin{array}{l}\text { Be grateful Gran Tangi: wees } \\
\text { dankbaar }\end{array}$ & $\begin{array}{l}\text { - Psycho-education on gratitude } \\
\text { - Relaxation, breathing exercise (i) } \\
\text { - Writing down a gratitude statement (i) } \\
\text { - Verbally expressing gratitude (g) } \\
\text { - Writing a gratitude letter (i) } \\
\text { - Closing moment: verbal expression of positive emotions (g) } \\
\text { - Home work (optional): gratitude visit (i), three good things (i) }\end{array}$ \\
\hline 2 & $\begin{array}{l}\text { Positivity starts with you Positiviteit } \\
\text { begint bij jezelf }\end{array}$ & $\begin{array}{l}\text { - Psycho-education on positive and negative emotions (i) } \\
\text { - Relaxation, breathing exercise (i) } \\
\text { - Reflection on homework assignments (g) } \\
\text { - Discovering and discussing positive and negative emotions (g) } \\
\text { - Outdoor random acts of kindness and presentation (g) } \\
\text { - Closing moment: verbal expression of positive emotions (g) } \\
\text { - Home work (optional): gratitude visit (i), three good things (i), } \\
\text { random acts of kindness at home or work (i) }\end{array}$ \\
\hline 3 & $\begin{array}{l}\text { Developing your own strengths } \\
\text { Bouw aan je sterke kanten }\end{array}$ & $\begin{array}{l}\text { - Psycho-education on strengths } \\
\text { - Relaxation, breathing exercise (i) } \\
\text { - Reflection on homework assignments (g) } \\
\text { - Discovering strengths (g) } \\
\text { - Creating a strength symbol/strength word cloud (g) } \\
\text { - Closing moment: verbal expression of positive emotions (g) } \\
\text { - Home work (optional): gratitude visit (i), three good things (i), } \\
\text { random acts of kindness at home or work, using strengths at } \\
\text { work or at home (i) }\end{array}$ \\
\hline 4 & $\begin{array}{l}\text { Your goals are attainable Jouw } \\
\text { doelen zijn haalbaar }\end{array}$ & $\begin{array}{l}\text { - Psycho-education on goal setting (SMART goals) } \\
\text { - Physical exercise (i) } \\
\text { - Reflection on homework assignments (g) } \\
\text { - Discussion on goal setting, Checking and developing a SMART } \\
\text { goal } \\
\text { - Closing moment: verbal expression of positive emotions (g) }\end{array}$ \\
\hline 5 & $\begin{array}{l}\text { Over coming your problems Je } \\
\text { problemen overwinnen }\end{array}$ & $\begin{array}{l}\text { - Psycho-education on Adversity/Belief and Consequences } \\
\text { - Written exercises in changing negative to positive beliefs } \\
\text { - Closing moment: verbal expression of positive emotions (g) }\end{array}$ \\
\hline 6 & Let it go Laat het los & $\begin{array}{l}\text { - Psycho-education on forgiveness and spirituality } \\
\text { - Moment of silence: exercise in thoughtless awareness (i) } \\
\text { - Forgiveness exercise (writing and verbal expression) (g/i) } \\
\text { - Expressing gratitude through prayer and surrendering (i) } \\
\text { - Closing moment: verbal expression of positive emotions (g) }\end{array}$ \\
\hline
\end{tabular}

(i): individual exercise; $(\mathrm{g})$ : group exercise 


\section{Appendix 2: Supplemental material: Moderator analysis}

Table 4 Baseline characteristics of participants in the intervention group, control group and the total sample

\begin{tabular}{|c|c|c|c|c|c|c|c|}
\hline & SMS $(n=80)$ & $\begin{array}{l}\text { WL } \\
(n=78)\end{array}$ & $\begin{array}{l}\text { Total } \\
(n=158)\end{array}$ & & $\begin{array}{l}\text { SMS } \\
(n=80)\end{array}$ & $\begin{array}{l}\text { WL } \\
(n=78)\end{array}$ & $\begin{array}{l}\text { Total } \\
(\mathrm{n}=158)\end{array}$ \\
\hline Age, M (SD) & $36.2(9.4)$ & $36.5(9.9)$ & $36.3(9.6)$ & \multicolumn{4}{|c|}{ Companies, $n(\%)$} \\
\hline Gender, $n(\%)$ & & & & InterMed & $35(44.9)$ & $35(43.8)$ & $70(44.3)$ \\
\hline Male & $38(48.8)$ & $25(32.1)$ & $63(39.9)$ & $S P S B$ & $28(35.9)$ & $17(21.3)$ & $45(28.5)$ \\
\hline Female & $42(51.2)$ & $53(67.9)$ & $95(60.1)$ & $M E S$ & $15(19.2)$ & $28(35.0)$ & $43(27.2)$ \\
\hline \multicolumn{8}{|l|}{ Ethnicity, $n(\%)$} \\
\hline Javanese & $39(48.8)$ & $27(34.6)$ & $66(41.8)$ & \multicolumn{4}{|c|}{ Education, $n(\%)$} \\
\hline Hindustani & $18(22.5)$ & $22(28.2)$ & $40(25.3)$ & Lower & $44(55.0)$ & $31(39.7)$ & $75(47.5)$ \\
\hline Afro-Surinamese & $6(7.5)$ & $18(23.1)$ & $24(15.2)$ & Middle & $29(36.3)$ & $36(46.2)$ & $65(41.1)$ \\
\hline Mixed & $17(21.3)$ & $10(12.8)$ & $27(17.1)$ & Higher & $7(8.8)$ & $11(14.1)$ & $18(11.4)$ \\
\hline Amerindian & $0(0.0)$ & $1(1.3)$ & $1(0.6)$ & & & & \\
\hline \multicolumn{4}{|c|}{ Monthly net salary in US dollars } & \multicolumn{4}{|l|}{ Religion, $n(\%)$} \\
\hline$<\$ 135$ & $18(22.5)$ & $13(16.7)$ & $31(19.6)$ & Christian & $30(37.5)$ & $36(46.2)$ & $66(41.8)$ \\
\hline$\$ 135-\$ 270$ & $37(46.3)$ & $35(44.9)$ & $72(45.6)$ & Islam & $24(30.0)$ & $23(29.5)$ & $47(29.7)$ \\
\hline$\$ 270-\$ 400$ & $11(13.8)$ & $13(16.7)$ & $24(15.2)$ & Hinduism & $15(18.8)$ & $14(17.9)$ & $29(18.4)$ \\
\hline$\$ 400-\$ 530$ & $9(11.3)$ & $10(12.8)$ & $19(12.0)$ & Javanism & $3(3.8)$ & $0(0.0)$ & $3(1.9)$ \\
\hline$>530$ & $5(6.3)$ & $7(9.0)$ & $12(7.6)$ & None & $8(10.0)$ & $5(6.4)$ & $13(8.2)$ \\
\hline \multicolumn{8}{|c|}{ Adherence, n (\%) } \\
\hline High & $46(57.5)$ & & & & & & \\
\hline Moderate & $17(21.3)$ & & & & & & \\
\hline Low & $17(21.3)$ & & & & & & \\
\hline
\end{tabular}

SMS = Strong Minds Suriname intervention group; WL = Wait-list control groupInterMed = Intermed Caribe N.V.; SPSB = Surinaamse Postspaarbank; MES = Multi-Electrical Systems N.V

Table 5 Moderator analyses of mental well-being (moderator x group interaction effects)

\begin{tabular}{|c|c|c|c|c|c|c|}
\hline \multirow[b]{2}{*}{ Demographic } & \multicolumn{3}{|c|}{$\mathrm{MHC}-\mathrm{SF}$ at post-test } & \multicolumn{3}{|c|}{$\mathrm{MHC}-\mathrm{SF}$ at 3-month follow-up } \\
\hline & $b$ & $95 \% \mathrm{CI}$ & $p$ & $b$ & $95 \% \mathrm{CI}$ & $p$ \\
\hline Age & .000 & $-.014-.014$ & .986 & -.010 & $-.024-.004$ & .154 \\
\hline Gender & .122 & $-.132-.376$ & .948 & -.137 & $-.386-.111$ & .276 \\
\hline Ethnicity & -.119 & $-.225--.012$ & $.030 *$ & .020 & $-.089-.127$ & .720 \\
\hline Religion & -.045 & $-.117-.027$ & .218 & -.047 & $-.112-.023$ & .187 \\
\hline Education & .133 & $-.070-.337$ & .197 & .050 & $-.152-.252$ & .626 \\
\hline Company & -.071 & $-.216-.073$ & .331 & -.067 & $-.210-.075$ & .354 \\
\hline Financial stress & .005 & $-.069-.079$ & .890 & .055 & $-.013-.123$ & .110 \\
\hline Adherence & .110 & $-.090-.303$ & .286 & -.001 & $-.199-.197$ & .991 \\
\hline
\end{tabular}

MHC-SF = Mental Health Continuum Short Form. B refers to the unique contribution of the interaction item (moderator $\mathrm{x}$ group) in the prediction of the RS-nl after controlling for the separate effects of group and moderator, and RS-nl at baseline

*significant at $95 \%$ CI 
Table 6 Moderator analyses of resilience (moderator x group interaction effects)

\begin{tabular}{|c|c|c|c|c|c|c|}
\hline \multirow[b]{2}{*}{ Demographic } & \multicolumn{3}{|c|}{ RS-nl at post-test } & \multicolumn{3}{|c|}{ RS-nl at 3-month follow-up } \\
\hline & $b$ & $95 \% \mathrm{CI}$ & $p$ & $b$ & $95 \% \mathrm{CI}$ & $p$ \\
\hline Age & -.002 & $-.02-0.110$ & .720 & -.011 & $-.024-.002$ & .101 \\
\hline Gender & .1290 & $-.134-.392$ & .334 & .032 & $-.225-.289$ & .806 \\
\hline Ethnicity & -.056 & $-.162-.050$ & .299 & -.006 & $-.101-.099$ & .918 \\
\hline Religion & -.033 & $-.099-.033$ & .322 & .007 & $-.057-.71$ & .827 \\
\hline Education & .207 & $-.005-.419$ & .059 & .028 & $-.181-.237$ & .792 \\
\hline Company & -.064 & $-.221-.094$ & .424 & -.030 & $-.184-.123$ & .697 \\
\hline Financial stress & -.169 & $-.049-.082$ & .613 & .071 & $-.001-.143$ & .054 \\
\hline Adherence & .031 & $-.135-.198$ & .708 & .038 & $-.137-.213$ & .426 \\
\hline
\end{tabular}

RS-nl = Dutch Resilience Scale. $\mathrm{B}$ refers to the unique contribution of the interaction item (moderator $\mathrm{x}$ group) in the prediction of the RS-nl after controlling for the separate effects of group and moderator, and RS-nl at baseline

\section{References}

Abbott, J. A., Klein, B., Hamilton, C., \& Rosenthal, A. (2009). The impact of online resilience training for sales managers on wellbeing and work performance. E-Journal of Applied Psychology, 5(1).

Abiola, T., \& Udofia, O. (2011). Psychometric assessment of the Wagnild and Young's resilience scale in Kano, Nigeria. BMC Research Notes, 4(1), 509.

Akhtar, S., \& Barlow, J. (2016). Forgiveness therapy for the promotion of mental well-being: A systematic review and meta-analysis. Trauma, Violence, \& Abuse, 1524838016637079.

Barron, R. M., \& Kenny, D. A. (1986). The moderator-mediator variable distinction in social psychological research: Conceptual, strategic, and statistical considerations. Journal of Personality and Social Psychology, 51(6), 1173-1182.

Ben-Itzhak, S., Bluvstein, I., \& Maor, M. (2014). The psychological flexibility questionnaire (PFQ): Development, reliability and validity. WebmedCentral PSYCHOLOGY 2014;5(4):WMC004606.

Block, J. (1961). Ego identity, role variability, and adjustment. Journal of Consulting Psychology, 25(5), 392.

Block, J., \& Block, J. H. (2006). Nursery school personality and political orientation two decades later. Journal of Research in Personality, 40(5), 734-749.

Bolier, L., Haverman, M., \& Walburg, J. A. (2010). Mental fitness: verbeter je mentale conditie. Amsterdam: Uitgeverij Boom.

Bolier, L., Haverman, M., Westerhof, G. J., Riper, H., Smit, F., \& Bohlmeijer, E. T. (2013). Positive psychology interventions: A meta-analysis of randomized controlled studies. BMC Public Health, 13, 119.

Buchanan, K. E., \& Bardi, A. (2010). Acts of kindness and acts of novelty affect life satisfaction. The Journal of Social Psychology, 150, 235-237.

Carr, A., Finnegan, L., Griffin, E., Cotter, P., \& Hyland, A. (2016). A randomized controlled trial of the say yes to life (SYTL) positive psychology group psychotherapy program for depression: An interim report. Journal of Contemporary Psychotherapy, 1-9.

Carver, C. S., \& Scheier, M. F. (2001). On the self-regulation of behavior. Cambridge: University Press. 
Chakhssi, F., Kraiss, J. T., Sommers-Spijkerman, M., \& Bohlmeijjer, E. T. (2018). The effect of positive psychology interventions on well-being in clinical populations: A systematic review and meta-analysis. BMC Psychiatry, 18(1), 211.

Cohn, M. A. (2008). Positive emotions: Short-term mechanisms, long-term outcomes, and mediating processes. University of Michigan.

Colijn, S., Snijders, H., \& Trijsburg, W. (2009). Universele therapiefactoren. In H. S. S. Colijn, M. Thunissen, S. Bogels, \& W. Trijsburg (Eds.), Leerboek psychotherapie (pp. 159-168). De Tijdstroom: Utrecht.

Crawford, J. R., \& Henry, J. D. (2004). The positive and negative affect schedule (PANAS): Construct validity, measurement properties and normative data in a large non-clinical sample. The British Journal of Clinical Psychology, 43(3), 245-265.

Cullen, B., Pownall, J., Cummings, J., Baylan, S., Broomfield, N., Haig, C., Kersel, D., Murray, H., \& Evans, J. J. (2015). Positive PsychoTherapy in ABI rehab (PoPsTAR): A pilot randomised controlled trial. Neuropsychological Rehabilitation, 28(1), 17-33.

Dempster, A. P., Laird, N. M., \& Rubin, D. B. (1977). Maximum likelihood from incomplete data via the EM algorithm. Journal of the Royal Statistical Society: Series B: Methodological, 1-38.

Domenech-Rodriguez, M., \& Wieling, E. (2004). Developing culturally appropriate, evidence-based treatments for interventions with ethnic minority populations. In M. R. E. Weiling (Ed.), Voices of color: First person accounts of ethnic minority therapists (pp. 313-333). Thousand Oaks: Sage.

Emmons, R. A., \& McCullough, M. E. (2003). Counting blessings versus burdens: An experimental investigation of gratitude and subjective well-being in daily life. Journal of Personality and Social Psychology, 84(2), 377-389.

Engelen, U., De Peuter, S., Victoir, A., Van Diest, I., \& Van den Bergh, O. (2006). Verdere validering van de Positive and Negative Affect Schedule (PANAS) en vergelijking van twee Nederlandstalige versies. Gedrag en Gezondheid, 34(2), 61-70.

Feicht, T., Wittmann, M., Jose, G., Mock, A., Hirschhausen, E. Von, \& Esch, T. (2013). Evaluation of a seven-week web-based happiness training to improve psychological well-being, reduce stress, and enhance mindfulness and flourishing: A randomized controlled occupational health study. EvidenceBased Complementary and Alternative Medicine, Article ID 676953.

Fledderus, M., Bohlmeijer, E. T., Fox, J.-P., Schreurs, K. M., \& Spinhoven, P. (2013). The role of psychological flexibility in a self-help acceptance and commitment therapy intervention for psychological distress in a randomized controlled trial. Behaviour Research and Therapy, 51(3), 142-151.

Frank, J. D., Frank, J., \& D. (1991). Persuasion and healing. Baltimore: John Hopkins University.

Fredrickson, B. L. (2001). The role of positive emotions in positive psychology: The broaden-and-build theory of positive emotions. The American Psychologist, 56(3), 218.

Fredrickson, B. L. (2004). The broaden-and-build theory of positive emotions. Philosophical Transactions of the Royal Society B: Biological Sciences, 359(1449), 1367.

Fredrickson, B. L., \& Branigan, C. (2005). Positive emotions broaden the scope of attention and thoughtaction repertoires. Cognition \& Emotion, 19(3), 313-332.

Fredrickson, B. L., Tugade, M. M., Waugh, C. E., \& Larkin, G. R. (2003). What good are positive emotions in crisis? A prospective study of resilience and emotions following the terrorist attacks on the United States on September 11th, 2001. Journal of Personality and Social Psychology, 84(2), 365.

Harzing, A.-W. (2006). Response styles in cross-national survey research: A 26-country study. International Journal of Cross-cultural Management, 6(2), 243-266.

Hayes, S. (2006). The six core processes of ACT. Contextual Science. org: ACBS.

Hayes, A. F. (2012). PROCESS: A versatile computational tool for observed variable mediation, moderation, and conditional process modeling. KS: University of Kansas.

He, J., \& van de Vijver, F. (2012). Bias and equivalence in cross-cultural research. Online Readings in Psychology and Culture, 2(2), 8.

He, J., Bartram, D., Inceoglu, I., \& Van de Vijver, F. J. (2014). Response styles and personality traits: A multilevel analysis. Journal of Cross-Cultural Psychology, 45(7), 1028-1045.

Heilemann, M. V., Lee, K., \& Kury, F. S. (2003). Psychometric properties of the Spanish version of the resilience scale. Journal of Nursing Measurement, 11(1), 61-72.

Hendriks, T., Graafsma, T., Hassankhan, A., Bohlmeijer, E., \& de Jong, J. (2017). Strengths and virtues and the development of resilience: A qualitative study in Suriname during a time of economic crisis. International Journal of Social Psychiatry, 0020764017749624.

Hendriks, T., Hassankhan, A., Schotanus-Dijkstra, M., Graafsma, T., Bohlmeijer, E. T., \& de Jong, J. (2018). The efficacy of non-Western positive psychology interventions: A meta-analysis and systematic review. International Journal of Wellbeing, 8(1), 71-98. 
Hendriks, T., Schotanus-Dijkstra, M., Hassankhan, A., Graafsma, T., de Jong, J., \& Bohlmeijer, E. T. (2019). The efficacy of multi-component positive psychology interventions: A meta-analysis. The Journal of Happiness Studies, https://doi.org/10.1007/s10902-10019-00082-10901.

Hendriks, T., Schotanus-Dijkstra, M., Hassankhan, A., Sardjo, W., Graafsma, T., Bohlmeijer, E., \& de Jong, J. (2020). Resilience and well-being in the Caribbean: Findings from a randomized controlled trial of a culturally adapted multi-component positive psychology intervention. The Journal of Positive Psychology, 15(2), 238-253.

Hinton, D. E., \& Jalal, B. (2014). Guidelines for the implementation of culturally sensitive cognitive behavioural therapy among refugees and in global contexts. Intervention, 12, 78-93.

Hinton, D. E., \& Kirmayer, L. J. (2016). The flexibility hypothesis of healing. Culture, Medicine and Psychiatry, 1-32.

Hinton, D. E., \& Kirmayer, L. J. (2017). The flexibility hypothesis of healing. Culture, Medicine, and Psychiatry, 41, 3-34.

Hinton, D. E., Rivera, E. I., Hofmann, S. G., Barlow, D. H., \& Otto, M. W. (2012). Adapting CBT for traumatized refugees and ethnic minority patients: Examples from culturally adapted CBT (CA-CBT). Transcultural Psychiatry, 49(2), 340-365.

Hinton, D. E., Pich, V., Hofmann, S. G., \& Otto, M. W. (2013). Acceptance and mindfulness techniques as applied to refugee and ethnic minority populations with PTSD: Examples from culturally adapted CBT. Cognitive and Behavioral Practice, 20(1), 33-46.

Isen, A. M. (1984). Toward understanding the role of affect in cognition. In R. S. Wyer Jr. \& T. K. Srull (Eds.), Handbook of social cognition (Vol. 3, pp. 179-236). Mahwah: Lawrence Erlbaum Associates Publishers.

Isen, A. M., Johnson, M. M., Mertz, E., \& Robinson, G. F. (1985). The influence of positive affect on the unusualness of word associations. Journal of Personality and Social Psychology, 48(6), 1413.

Isen, A. M., Daubman, K. A., \& Nowicki, G. P. (1987). Positive affect facilitates creative problem solving. Journal of Personality and Social Psychology, 52(6), 1122.

Joshanloo, M. (2016). A new look at the factor structure of the MHC-SF in Iran and the United States using exploratory structural equation modeling. Journal of Clinical Psychology, 72(7), 701-713.

Kashdan, T. B., \& Rottenberg, J. (2010). Psychological flexibility as a fundamental aspect of health. Clinical Psychology Review, 30(7), 865-878.

Kashdan, T. B., Barrios, V., Forsyth, J. P., \& Steger, M. F. (2006). Experiential avoidance as a generalized psychological vulnerability: Comparisons with coping and emotion regulation strategies. Behaviour Research and Therapy, 44(9), 1301-1320.

Kazdin, A. E. (2007). Mediators and mechanisms of change in psychotherapy research. Annual Review of Clinical Psychology, 3, 1-27.

Kazdin, A. E. (2009). Understanding how and why psychotherapy leads to change. Psychotherapy Research, $19(4-5), 418-428$.

Keyes, C. L. M. (2005). Mental illness and/or mental health? Investigating axioms of the complete, state model of health. Journal of Consulting and Clinical Psychology, 73, 539-554.

Keyes, C. L., Wissing, M., Potgieter, J. P., Temane, M., Kruger, A., \& Van Rooy, S. (2008). Evaluation of the mental health continuum-short form (MHC-SF) in setswana-speaking south Africans. Clinical Psychology \& Psychotherapy, 15(3), 181-192.

KNOEMA (May 4, 2018). Suriname - average consumer prices inflation rate. World data atlas.Retrieved from https://knoema.com/atlas/Suriname/Inflation-rate.

Kraemer, H. C., Wilson, G. T., Fairburn, C. G., \& Agras, W. S. (2002). Mediators and moderators of treatment effects in randomized clinical trials. Archives of General Psychiatry, 59(10), 877-883.

Lamers, S., Westerhof, G. J., Bohlmeijer, E. T., ten Klooster, P. M., \& Keyes, C. L. (2011). Evaluating the psychometric properties of the mental health continuum-short form (MHC-SF). Journal of Clinical Psychology, 67(1), 99-110.

Lee, S. T., Hartanto, A., Yong, J. C., Koh, B., \& Leung, A. K. y. (2020). Examining the cross-cultural validity of the positive affect and negative affect schedule between an Asian (Singaporean) sample and a Western (American) sample. Asian Journal of Social Psychology, 23, 109-116.

Li, M., Jiang, X., \& Ren, Y. (2017). Mediatior effects of positive emotions on social support and depression among adolescents suffering from mobile phone addiction. Psychiatria Danubina, 29(2), 207-213.

Lim, Y. J. (2014). Psychometric characteristics of the Korean mental health continuum-short form in an adolescent sample. Journal of Psychoeducational Assessment, 32(4), 356-364.

Lyubomirsky, S., \& Layous, K. (2013). How do simple positive activities increase well-being? Current Directions in Psychological Science, 22(1), 57-62. 
Munasinge, K. (2012). Adaptation and validation of the resilience scale on Sinhala speaking adolescents attending school in the Kandy District. In Paper presented at the annual research symposium 2012. Colombo: University of Colombo.

Nikrahan, G. R., Laferton, J. A., Asgari, K., Kalantari, M., Abedi, M. R., Etesampour, A., . . Huffman, J. C. (2016). Effects of positive psychology interventions on risk biomarkers in coronary patients: A randomized, wait-list controlled pilot trial. Psychosomatics: Journal of.

Ong, A. D., Bergeman, C. S., Bisconti, T. L., \& Wallace, K. A. (2006). Psychological resilience, positive emotions, and successful adaptation to stress in later life. Journal of Personality and Social Psychology, 91(4), 730.

Otto, A. K., Szczesny, E. C., Soriano, E. C., Laurenceau, J.-P., \& Siegel, S. D. (2016). Effects of a randomized gratitude intervention on death-related fear of recurrence in breast cancer survivors. Health Psychology, 35(12), 1320-1328.

Patterson, C. M., \& Newman, J. P. (1993). Reflectivity and learning from aversive events: Toward a psychological mechanism for the syndromes of disinhibition. Psychological Review, 100(4), 716.

Perugini, M. L. L., de la Iglesia, G., Solano, A. C., \& Keyes, C. L. M. (2017). The mental health continuumshort form (MHC-SF) in the ARGENTinean context: Confirmatory factor analysis and measurement invariance. Europe's Journal of Psychology, 13(1), 93.

Pesce, R., Assis, S., Avanci, J., Santos, N., Malaquias, J., \& Carvalhaes, R. (2005). Cross-cultural adaptation, reliability and validity of the resilience scale. Cadernos de saude publica, 21(2), 436-448.

Portzky, M., Wagnild, G., De Bacquer, D., \& Audenaert, K. (2010). Psychometric evaluation of the Dutch resilience scale RS-nl on 3265 healthy participants: A confirmation of the association between age and resilience found with the Swedish version. Scandinavian Journal of Caring Sciences, 24(s1), 86-92.

Prawitz, A. D., Garman, E. T., Sorhaindo, B., O'Neill, B., Kim, J., \& Drentea, P. (2006). InCharge financial distress/financial well-being scale: Development, administration, and score interpretation. Journal of Financial Counseling and Planning, 17(1), 34-50.

Preacher, K. J., \& Hayes, A. F. (2008). Asymptotic and resampling strategies for assessing and comparing indirect effects in multiple mediator models. Behavior Research Methods, 40(3), 879-891.

Proctor, C., Maltby, J., \& Linley, P. A. (2011). Strengths use as a predictor of well-being and health-related quality of life. Journal of Happiness Studies, 12(1), 153-169.

Proyer, R. T., Gander, F., Wellenzohn, S., \& Ruch, W. (2015). Strengths-based positive psychology interventions: a randomized placebo-controlled online trial on long-term effects for a signature strengths- vs a lesser strengths-intervention. Frontiers in Psychology, 6, 456.

Proyer, R. T., Gander, F., Wellenzohn, S., \& Ruch, W. (2016). Addressing the role of personality, ability, and positive and negative affect in positive psychology interventions: Findings from a randomized intervention based on the authentic happiness theory and extensions. The Journal of Positive Psychology, 11(6), 609-621.

Quoidbach, J., Mikolajczak, M., \& Gross, J. J. (2015). Positive interventions: An emotion regulation perspective. Psychological Bulletin, 141(3), 655.

Robertson, I. T., Cooper, C. L., Sarkar, M., \& Curran, T. (2015). Resilience training in the workplace from 2003 to 2014: A systematic review. Journal of Occupational and Organizational Psychology, 88(3), 533-562.

Rusk, R. D., \& Waters, L. E. (2013). Tracing the size, reach, impact, and breadth of positive psychology. The Journal of Positive Psychology, 8(3), 207-221.

Rusk, R. D., Vella-Brodrick, D. A., \& Waters, L. (2018). A complex dynamic systems approach to lasting positive change: The synergistic change model. The Journal of Positive Psychology, 13(4), 406-418.

Sandage, S. J., \& Worthington Jr., E. L. (2010). Comparison of two group interventions to promote forgiveness: Empathy as a mediator of change. Journal of Mental Health Counseling, 32(1), 35-57.

Schiffrin, H. H. (2014). Positive psychology and attachment: Positive affect as a mediator of developmental outcomes. Journal of Child and Family Studies, 23(6), 1062-1072.

Schotanus-Dijkstra, M., Pieterse, M. E., Drossaert, C. H., Walburg, J. A., \& Bohlmeijer, E. T. (2019). Possible mechanisms in a multicomponent email guided positive psychology intervention to improve mental wellbeing, anxiety and depression: A multiple mediation model. The Journal of Positive Psychology, 14(2), $141-155$.

Schueller, S. M., \& Parks, A. C. (2014). The science of self-help. European Psychiatry, 19(2), 145-155.

Schueller, S., Kashdan, T., \& Parks, A. (2014). Synthesizing positive psychological interventions: Suggestions for conducting and interpreting meta-analyses. International Journal of Wellbeing, 4(1).

Seligman, M. E. P., Rashid, T., \& Parks, A. C. (2006). Positive psychotherapy. The American Psychologist, $61(8), 774$ 
Sheldon, K. M., \& Lyubomirsky, S. (2012). The challenge of staying happier: Testing the hedonic adaptation prevention model. Personality and Social Psychology Bulletin, 38(5), 670-680.

Shin, L. J., \& Lyubomirsky, S. (2017). Increasing well-being in independent and interdependent cultures. In M. A. Warren \& .S. I. Donaldson, Scientific advances in positive psychology (pp. 11-36). Santa Barbara: Praeger.

Sin, N. L., \& Lyubomirsky, S. (2009). Enhancing well-being and alleviating depressive symptoms with positive psychology interventions: A practice-friendly meta-analysis. Journal of Clinical Psychology, 65, $467-487$.

Sood, A., Prasad, K., Schroeder, D., \& Varkey, P. (2011). Stress management and resilience training among Department of Medicine faculty: A pilot randomized clinical trial. Journal of General Internal Medicine, 26(8), 858-861.

Toussaint, L., Barry, M., Bornfriend, L., \& Markman, M. (2014). Restore: The journey toward self-forgiveness: A randomized trial of patient education on self-forgiveness in Cancer patients and caregivers. Journal of Health Care Chaplaincy, 20(2), 54-74.

Tsai, J. L., Knutson, B., \& Fung, H. H. (2006). Cultural variation in affect valuation. Journal of Personality and Social Psychology, 90(2), 288.

Tugade, M. M., \& Fredrickson, B. L. (2004). Resilient individuals use positive emotions to bounce back from negative emotional experiences. Journal of Personality and Social Psychology, 86(2), 320.

Vulpe, A., \& Dafinoiu, I. (2012). Positive emotions, coping strategies and ego-resiliency: A mediation model. Procedia-Social and Behavioral Sciences, 33, 308-312.

Wagnild, G., \& Young, H. (1993). Development and psychometric. Journal of Nursing Measurement, 1(2), $165-178$.

Watson, D., Clark, L. A., \& Tellegen, A. (1988). Development and validation of brief measures of positive and negative affect: The PANAS scales. Journal of Personality and Social Psychology, 54(6), 10631070 .

Weidong, Z., Jing, D., \& Schick, C. J. (2004). The cross-cultural measurement of positive and negative affect examining the dimensionality of PANAS. Psychological Science (China), 27(1), 77-79.

Wicksell, R. K., Olsson, G. L., \& Hayes, S. C. (2010). Psychological flexibility as a mediator of improvement in acceptance and commitment therapy for patients with chronic pain following whiplash. European Journal of Pain, 14(10), 1059-e1051-1059. e1011.

Yang, Y., Li, M.-H., \& Xia, Y. (2012). Measurement invariance of the resilience scale. The International Journal of Educational and Psychological Assessment, 11(2), 1-19.

Yu, N., Lam, T., Liu, I. K., \& Stewart, S. M. (2015). Mediation of short and longer term effects of an intervention program to enhance resilience in immigrants from mainland China to Hong Kong. Frontiers in Psychology, 6.

Publisher's Note Springer Nature remains neutral with regard to jurisdictional claims in published maps and institutional affiliations.

\section{Affiliations}

\section{Tom Hendriks ${ }^{1} \cdot$ Marijke Schotanus-Dijkstra $^{2} \cdot$ Tobi Graafsma $^{3} \cdot$ Ernst Bohlmeijer $^{4}$. Joop de Jong ${ }^{5}$}

1 Faculty of Social Sciences, Department of Psychology, Anton de Kom Universiteit van Suriname, Leysweg 86, P.O.B. 9212, Paramaribo, Suriname

2 Department of Psychology, Health and Technology, University of Twente, Centre for eHealth and Wellbeing Research (CEWR), Enschede, The Netherlands

3 Institute of Graduate Studies and Studies (IGSR), Anton de Kom University of Suriname, Paramaribo, Suriname

4 Department of Psychology, Health and Technology Centre for eHealth and Wellbeing Research (CEWR), University of Twente, Enschede, The Netherlands

5 Amsterdam University Medical Center, Amsterdam, the Netherlands 